\title{
Automated 3D Segmentation of the Lung Airway Tree Using Gain-Based Region Growing Approach
}

\author{
Harbir Singh $^{1}$, Michael Crawford, ${ }^{2}$, John Curtin ${ }^{2}$, and Reyer Zwiggelaar ${ }^{1}$ \\ 1 School of Computing Sciences, University of East Anglia, Norwich NR4 7TJ, England. \\ \{Harbir.Singh, R.Zwiggelaar\}@uea.ac.uk \\ 2 Norfolk and Norwich University Hospital, Norwich NR4 7UY, England. \\ \{Michael.Crawford, John.Curtin\}@nnuh.nhs.uk
}

\begin{abstract}
In diagnosing lung diseases, it is highly desirable to be able to segment the lung into physiological structures, such as the intra-thoracic airway tree and the pulmonary structure. Providing an in-vivo and non-invasive tool for 3D reconstruction of anatomical tree structures such as the bronchial tree from 2D and 3D data acquisitions is a challenging issue for computer vision in medical imaging. Due to the complexity of the tracheobronchial tree, the segmentation task is non trivial. This paper describes a 3D adaptive region growing algorithm incorporating gain calculation for segmenting the primary airway tree using a stack of $2 \mathrm{D}$ CT slices. The algorithm uses an entropy-based measure known as information gain as a heuristic for selecting the voxels that are most likely to represent the airway regions.
\end{abstract}

\section{Introduction}

In diagnosing lung diseases, it is essential to be able to segment the lung into physiological structures, such as the intra-thoracic airway tree and the pulmonary structure. Once the airway tree is extracted, quantitative analysis can be performed to evaluate the tree structure and function. The tree structure is important in examining physiologic and pathological conditions such as stenosis and tumors. Given the complex branching of the airway tree, it is challenging to segment the tree manually. Such a manual annotation process is not free of problems. Image quality is compromised due to CT image acquisition process leading to intensity inhomogeneity. Partial volume effects make it difficult to identify small airways and minor detail clearly at the lower end of the tree. If the airway direction is parallel to the image plane, the density values in the airway lumen change, making the tracking of the airway regions difficult based on their intensity profile [1].

A number of automated approaches exist, covering segmentation and skeletonisation algorithms to extract all major airway branches and large parts of minor distal branches. Some of the approaches are based on mathematical morphology [2], central axis analysis [3], region growing [4], and knowledge-based techniques [5]. There is a trend towards hybrid approaches using a combination of techniques to give better results in terms of speed and detail of the extraction process [6]. A combination of morphological filtering, connection cost-based marking and a conditional watershed approach has been provided 
in [7] with use of fractal analysis and reconstruction. Other techniques are based on front propagation [8] to extract the major airway branches and parts of minor distal branches.

While 3D region growing is efficient, it suffers from partial volume effects, noise and global thresholding. The optimal threshold differs for large versus small airways, resulting in lack of finer details of the airways leading to incomplete structures and rough edges [6]. Similarly for method based on central axis analysis, selection of initial parameters, stopping criteria and forming of paths outside the airways warrants attention. For techniques based on morphology, the use and variation in morphological operators causes variation in the results obtained.

We have investigated the use of gain heuristic in 3D region growing for extracting the airway tree from a stack of volumetric CT data. The method has been evaluated against manually segmented tree obtained from the same data used for the automatic 3D method. The remainder of the paper is organized as follows. Section 2 explains the data and methods. Section 3 discusses the results and findings. Section 4 concludes with a few remarks and future directions.

\section{Materials and Methods}

\subsection{Data}

For each patient the data comprised of 125 transversal DICOM CT scans of $512 \times 512$ dimension obtained from Norfolk and Norwich University Hospital. The CT images are calibrated such that pure air voxel is represented by a value of about -1000 Hounsfield Units (HU), water is at $0 \mathrm{HU}$, soft tissue such as in the mediastinum is in the range of $-100 \mathrm{HU}$ to $200 \mathrm{HU}$ and bone is represented by a value of $+1000 \mathrm{HU}$ [6]. These files were reduced to $256 \times 256 \times 125$ stack of images using lung window level settings. Fig. 1] shows image slices at the top, middle and lower sections of the lung cavity. In these images dark grey levels correspond to low density zones and light grey levels correspond to high density zones respectively. Bronchi appear as dark zones with bronchial lumen surrounded by medium-high thin grey level zones called bronchial walls.

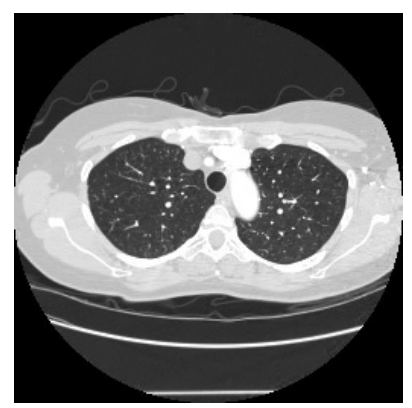

(a)

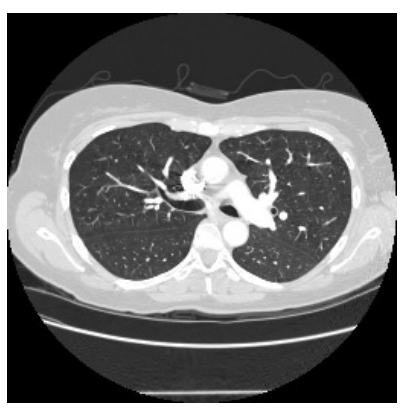

(b)

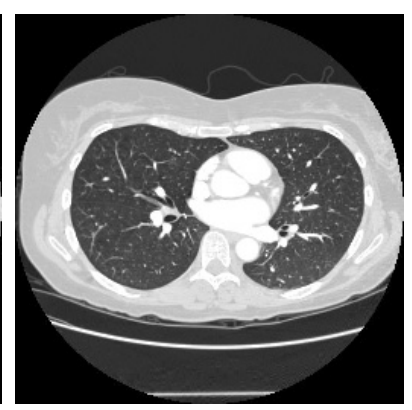

(c)

Fig. 1. Reconstructed transversal slices, where (a) CT slice showing the trachea, (b) branched airways at mid-section, and (c) airways in the lower-section. 


\subsection{Bronchus Structure}

The bronchus has a pipe structure with air inside. Beginning from the end of the trachea, it extends into the lungs branching repeatedly like a tree. The splitting mainly occurs in bifurcations where the parent branch splits into two child branches with decreased diameters and lengths. The bronchi are classified into lobar bronchi which supply the five lobes, the segmental bronchi and the sub-segmental bronchi [8]. The contrast of the bronchus image is low and the bronchi is surrounded by a thin wall with inner area filled with air. This wall gets thinner as the branching increases and diameter of the bronchi decreases. The bronchus appears as a circular ring when it cuts orthogonal through a slice. CT values are lower inside the bronchus than those on the surrounding bronchus walls. This enables extraction of the bronchus area by following those areas in which the CT value is low and surrounded by walls of relatively higher CT values [4]. However, as the bronchi walls of the sub-segmental bronchi become very thin, the partial volume effect and image artifacts can break up the wall structure causing problems in the segmentation of the bronchi.

An example of manual segmentation of the airway tree can be found in Fig. 2

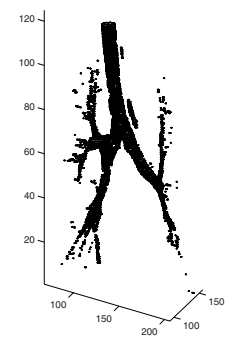

Fig. 2. Manual airway tree segmentation example.

\subsection{D Gain-Based Segmentation}

The 3D segmentation method is based on regions growing using $\mathrm{N}_{26}$ voxel connectivity and incorporates information gain heuristic at the voxel processing stage. The details of the methods are given below.

At each candidate voxel, the gain at the voxels in the $\mathrm{N}_{26}$ neighborhood is calculated. The voxels with information gain higher than the threshold gain, are chosen for inclusion in the region growing process. These voxels with the highest information gain below the threshold gain, represent the greatest entropy reduction at the candidate voxel and reflect the least randomness or impurity at the candidate voxel [9].

Let set $S$ consist of $n s$ data points in the neighborhood of candidate voxel $V$. The intensity value of each voxel is $I\left(x_{r}, y_{r}, z_{r}\right)$. Considering $\mathrm{N}_{26}$ neighborhood, our sample $S$, will consist of 27 points. A voxel can be included in the region growing process or it 
can be excluded. Hence there are two classes which the sample points will be classified into. If $I$ of each voxel is less than the threshold $T$, then that voxel is assigned a class of inclusion and if $I$ of a voxel is more than the threshold $T$, then that voxel is assigned a class of exclusion. Given that our class label $C$ has two values, $(C=$ include or $C=$ exclude $)$, let $s$ be the number of points belonging to class $C_{i}$ in our sample.

The expected information for the whole sample $S$, is obtained as

$$
E I\left(s_{1}, s_{2}, \ldots s_{m}\right)=-\sum_{i=1}^{m} p_{i} \log _{2}\left(p_{i}\right)
$$

where $\mathrm{p}_{i}$ is the probability of occurrence of voxel $s_{i}$ with class $\mathrm{C}_{i}$ in the arbitrary sample and is given by $s_{i} / n s$.

The expected information $E$, at a given sample point $s_{i}$ is given as

$$
E\left(s_{i}\right)=p\left(s_{i}\right) * E I\left(s_{i}\right)
$$

where $\mathrm{p}\left(\mathrm{s}_{i}\right)$ is the probability of sample point $\mathrm{s}_{i}$ in the sample.

Total gain is defined as

$$
G=E I\left(s_{1}, s_{2} \ldots s_{m}\right)-E\left(s_{i}\right)
$$

The voxel with a gain higher than the threshold gain, is included in the region growing process. Fig. 3 shows the intensity profile of the airway region varies due to intensity homogeneity artifacts. This variation affects the $3 \mathrm{D}$ region growing algorithm based on a fixed threshold. Using a normal 3D segmentation approach leads to a large amount of variation in the area that gets included in the region growing process leading to an inaccurate segmentation. Often an adaptive thresholding approach is used where the threshold is dynamically adjusted in the region growing process as followed in [6, [10]. We introduced a gain parameter to control the $3 \mathrm{D}$ region growing process. In our approach no pre-processing such as smoothing or extraction of lung area was used. The results depend on the resolution and the quality of the underlying dataset.

\subsection{Algorithm Outline}

The steps of the algorithm are outlined below.

1. Initialize the algorithm providing a seed point $S\left(x_{r}, y_{r}, z_{r}\right)$, threshold value $T$, and a gain value $G$.

2. Perform $3 \mathrm{D}$ region growing using $\mathrm{N}_{26}$ connectivity with the seed $S\left(x_{r}, y_{r}, z_{r}\right)$, having intensity $I\left(x_{r}, y_{r}, z_{r}\right)$

3. For all voxels in the $\mathrm{N}_{26}$ neighborhood of seed point $S$, with intensity below threshold $T$, include in the region growing process.

4. For all voxels in the $\mathrm{N}_{26}$ neighborhood of seed point $S$, with intensity above threshold $T$, and intensity below an upper limit of the threshold $T$ multiplied by a factor $K$, calculate gain $G$. $(K=1.1 \times T)$

5. If the calculated gain $G$ at the voxel is greater than the threshold gain $G$ provided, include this voxel in the region growing process else ignore the voxel.

6. Goto step 3 until all voxels have been processed.

7. The output is a 3D binary subvolume of segmented airway tree. 


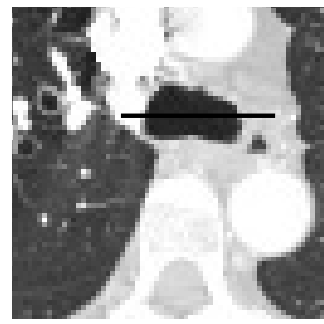

(a)

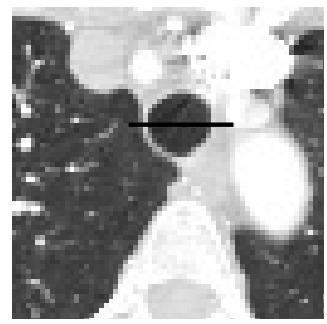

(c)

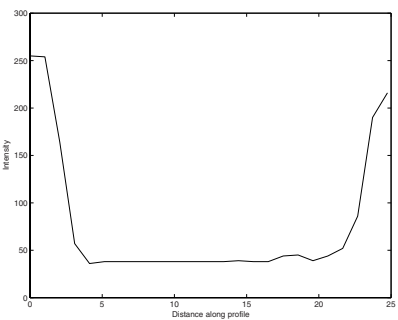

(b)

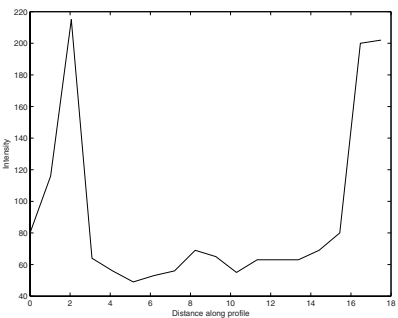

(d)

Fig. 3. (a,c) CT slice showing a bronchus and (b,d) the intensity profile across the bronchus.

\section{Results}

In this section we present and discuss the results obtained using the modified heuristic gain based 3D region growing algorithm.

Fig. 4 shows the processing results using $2 \mathrm{D}$ slices representing the top, middle and lower section of the bronchial tree. The 3D algorithm successfully identifies the bronchus area. Fig. $4 \mathrm{a}$ shows part of the trachea, Fig. $4 \mathrm{~b}$ shows the trachea before splitting and Fig. 4 r shows the airways when the trachea has split.

Fig. 5. shows the 3D visualization of the airway tree from the top, side and front angle segmented (threshold level equal to 53 and gain factor equal to 50\%). The algorithm has been successful in identifying the branching and the structure of the airway tree on the given dataset to a high degree of accuracy.

We undertook a more detailed analysis, based on a single dataset, of sensitivity and specificity by plotting the values at different gain settings for different levels of thresholds, which can be found in Fig. 6. The automated results are compared to the manually segmented tree. It should be noted that the current evaluation methodology has a bias towards the initial levels in the airway tree as these contain relatively more pixels than the final levels of the tree. A level dependant evaluation is part of our future research directions. We see that in Fig. 6a sensitivity for $\mathrm{T}=47$ is very low and in Fig. 6b specificity for $\mathrm{T}=47$ very high (specificity=1) implying that the threshold setting was too low for the automated method to pick out the airway region. For these low threshold values the results are an under-segmentation of the tree. For higher levels of thresholds, the sensitivity is higher, with sensitivity $=1$ for $\mathrm{T}=60,63$, while at the same level of thresholds, the specificity decreases as evident in Fig. 6b where the specificity curves shift downwards. This indicates an over-segmentation at these high threshold values. 

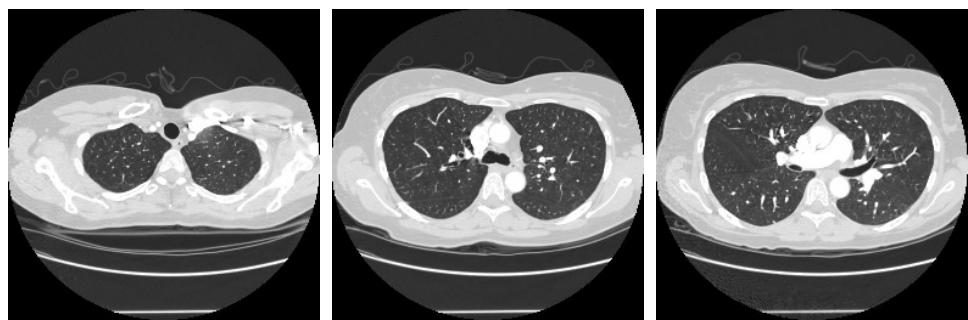

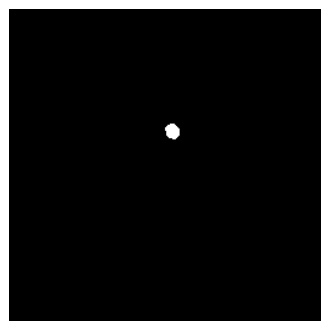

(a)

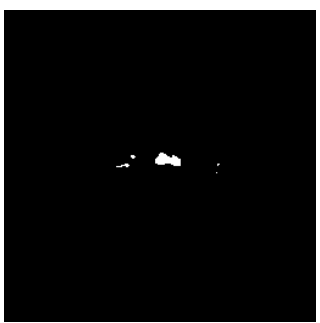

(b)

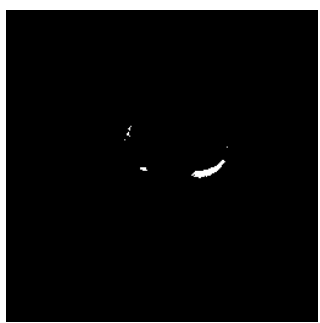

(c)

Fig. 4. 2D processing results showing original images and extracted bronchus area.

Using $\left(\min \left((1-\text { sensitivity })^{2}+(1-\text { specificity })^{2}\right)\right)$, which takes both the sensitivity and specificity into account, the overall optimal segmentation parameters, for this dataset, are $\mathrm{T}=57$ and $\mathrm{G}=70 \%$. The segmentation is robust with respect to both parameters as all results for the ranges $\mathrm{T}=53-60$ and $\mathrm{G}=10 \%-80 \%$ give acceptable results.

With respect to an increase in gain settings, Fig. 6a show almost constant sensitivity curves, with a slight decrease in sensitivity when the gain increases. However, Fig. $6 \mathrm{~b}$ shows that the specificity gradually increases when the gain is increased. This implies that the use of gain heuristic has a larger influence on the specificity than on sensitivity.

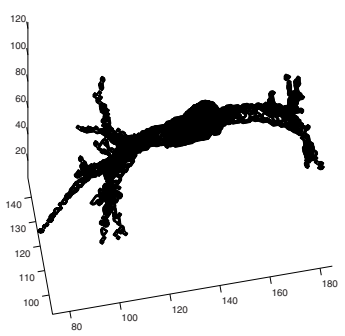

(a)

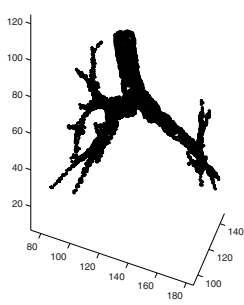

(b)

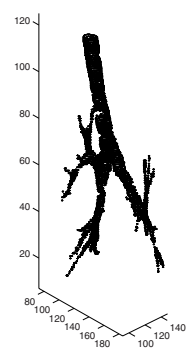

(c)

Fig. 5. Visualization of airway trees at $\mathrm{T}=53, \mathrm{G}=50 \%$, displaying (a) top view, (b) side view, and (c) front view. 


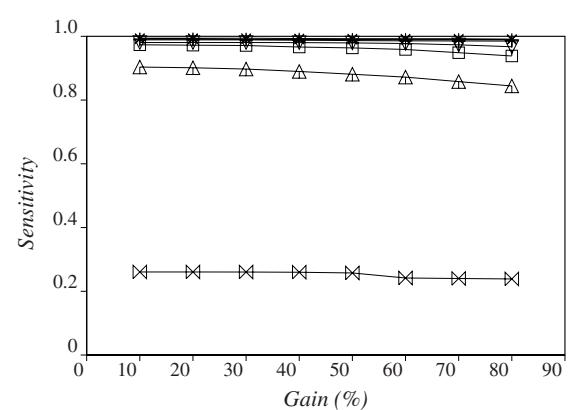

(a)

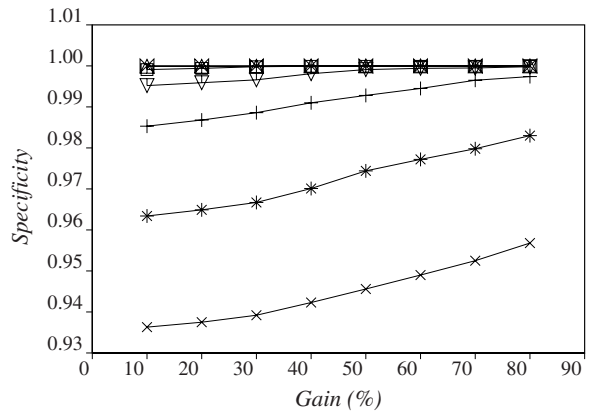

(b)

Fig. 6. Sensitivity and specificity graphs based on the following threshold values; $\bowtie: 47, \triangle: 50$, $\square: 53, \nabla: 55,+: 57, *: 60$ and $\times: 63$ )

\subsection{Limitations}

However, as seen in Fig. 7, the algorithm is not fail proof as it encounters the problem of leakage which affects all segmentations of the airway tree based on the region growing approach. Even though the heuristic gain was used in this case, it was not able to prevent leakage of the grow process into the lung parenchyma. The heuristic gain at best introduces an additional control facility in the extraction process and combined with other parameters such as explosion of volume and other features of the regions of interest [11], can help in achieving better segmentation results.

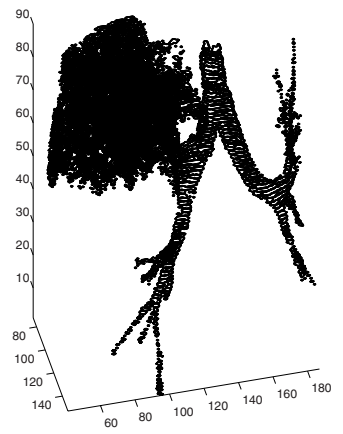

Fig. 7. Leakage, at $\mathrm{T}=63, \mathrm{G}=10 \%$, of the region grow process into the lung parenchyma.

\section{Conclusion}

The segmented bronchus area gives a measure of the volume of air in the lungs and can be subtracted from total lung volume to gauge a better estimate of volume of lung 
tissue. Accurate segmentation of the bronchus is also vital for applications like virtual bronchoscopy.

We have segmented the airway tree using a heuristic gain measure in a $3 \mathrm{D}$ region growing approach. Our results show a close correlation with manual segmentation and establish the use of gain as a reliable measure. A comparison of information gain based unsupervised image segmentation with alternate techniques can be found in [12].

Currently, our research concentrates on the development of a modified approach, which uses extracted features like the length of the branch, angles between branches and radius of the bronchus. We also intend to undertake a more robust evaluation on a larger number of patient datasets. This should aid in developing a normal lung model by efficiently modelling the airway tree topology and classification of cancerous regions.

\section{References}

1. Sonka, M., Fitzpatrick, J., eds.: Pulmonary Imaging and Analysis, Handbook Of Medical Imaging:Medical Image Processing and Analysis. Volume 2. SPIE - The International Society for Optical Engineering, Bellingham, Washington (2000)

2. Aykac, D., Hoffman, E., McLennan, G., Reinhardt, J.: Segmentation and analysis of the human airway tree from three-dimensional X-ray CT images. IEEE Transactions on Medical Imaging 22 (2003) 940-950

3. Swift, R., Kiraly, A., Sherbondy, A., Austin, A., Hoffman, E., McLennan, G., Higgins, W.: Automatic axes-generation for virtual bronchoscopic assessment of major airway obstructions. Computerized Medical Imaging and Graphics 26 (2002) 103-118

4. Mori, K., Hasegwawa, J., Toriwaki, J., Anno, H., Katada, K.: Automated extraction and visualisation of bronchus from 3D CT images of lung. In: CVRMed. Volume 905 of Lecture Notes in Computer Science. (1995) 542-548

5. Sonka, M., Park, W., Hoffman, E.: Rule-based detection of intrathoracic airway trees. IEEE Transactions on Medical Imaging 15 (1996) 314-326

6. Kiraly, A., Higgins, W., Hoffman, E., McLennan, G., Reinhardt, J.: 3D human airway segmentation for virtual bronchoscopy. In: Physiology and Function from Multidimensional Images. Volume 4683 of SPIE Medical Imaging. (2002) 16-29

7. Fetita, C., Preteux, F.: Three-dimensional reconstruction of human bronchial tree in HRCT. In: Proceedings SPIE Conference on Nonlinear Image Processing X. Volume 3646 of SPIE Electronic Imaging. (1999) 281-295

8. Schlatholter, T., Lorenz, C., Carlsen, I., Renisch, S., Deschamps, T.: Simultaneous segmentation and tree reconstruction of the airways for virtual bronchoscopy. In: Image Processing. Volume 4684 of SPIE Medical Imaging. (2002) 103-113

9. Han, J., Kamber, M.: Data Mining Concepts and Techniques. Morgan Kaufmann, San Francisco CA (2001)

10. Tschirren, J., Palagyi, K., Reinhardt, J., Hoffman, E., Sonka, M.: Segmentation, skeletonization and branchpoint matching - a fully automated quantitative evaluation of human intrathoracic airway trees. In: Medical Image Computing and Computer-Assisted Intervention,Part II. Volume 2489 of Lecture Notes in Computer Science. (2002) 12-19

11. Revol-Muller, C., Peyrin, F., Carrillon, Y., Odet, C.: Automated 3D region growing algorithm based on assessment function. Pattern Recognition Letters 23 (2002) 137

12. Singh, H., Zwiggelaar, R.: Unsupervised image segmentation using information gain. The Irish Machine Vision and Image Processing Conference (submitted) (2004) 